\title{
THREE YEARS PROSPECTIVE STUDY OF NECROTIZING ENTEROCOLITIS
}

\author{
Jain $\mathbf{N}^{*}$, Mangal V*
}

\begin{abstract}
Necrotizing Enterocolitis (NEC) is a major cause of mortality and morbidity in the newborn period. To know the incidence of NEC at our Neonatal Intensive Care Unit (NICU). Over a 3 year period (Prospective study) ,510 cases were studied for NEC. Incidence was 5.3\%, mortality due to NEC was $22 \%$, Asphyxia, Prematurity and enteral feeding were the common risk factor. Abdominal distention, blood in stool and air in intestinal wall were the main clinical features. Prevention and high index of suspicion are important to limit the morbidity and mortality.
\end{abstract}

\section{Key Words: Necrotizing enterocolitis, Low birth weight.}

\section{INTRODUCTION}

Necrotizing enterocolitis (NEC) is an acquired condition of diffuse necrotic injury to the mucosal and submucosal layers of the bowel. It is the most serious gastrointestinal (GI) disorder that occurs during the neonatal period. ${ }^{1-3}$ The entire GI tract, from the stomach to the anus, is susceptible. The distal small gut and proximal colon are involved most frequently. The lesions may be diffuse and contiguous or patchy and more focal in nature. Systemic signs and symptoms accompany GI injury.

Today NEC ranks second only to respiratory distress syndrome as a cause of Neonatal death in the west. ${ }^{4}$ Its incidence is variable among different centers. The Overall incidence varies between 1-2 per 1000 live births or $2-5 \%$ of admissions to the NICU. ${ }^{5,6}$

NEC is characterized by Gastrointestinal and systemic symptoms, feed intolerance, delayed gastric emptying, vomiting and abdominal distention, occult or gross bloody stools associated with systemic signs like lethargy, apnea, respiratory distress and poor perfusion, leading to bacteremia, shock ,acidosis and DIC. ${ }^{7}$

According to the modified classification the staging of NEC is done in 3 stages (on the basis of clinical and Radiological Picture). ${ }^{8}$

\section{Stage-1:}

Suspected NEC is associated with non specific clinical and radiological signs like temperature instability, mild abdominal distention, dilated bowel loops and bowel wall thickening on X-ray.

\section{Statge-2 :}

Definite NEC is characterized by gross blood in stool, mild metabolic acidosis and thrombocytopenia along with radiological finding of intestinal pneumatosis.

\footnotetext{
${ }^{*}$ Nepalgunj Medical College, B. P. Chowk, Nepalgunj, Nepal.

Address for correspondence : Dr. Neeraj Jain

Dept. of Padiatrics

Nepalgunj Medical College

B. P. Chowk, Nepalgunj, Nepal.

Email: ishu12002@yahoo.com
} 


\section{Stage-3 :}

Advanced NEC is characterized by hypotension, DIC and some times signs of generalized peritonitis

\section{OBJECTIVE}

There is paucity of published data about the incidence of NEC in Nepal. Therefore we planned to conduct this study to know the incidence and out come of NEC at our centre. We also studied the clinical picture of the NEC cases.

\section{MATERIAL AND METHODS}

This prospective study was conducted in the Neonatal intensive care unit of Nepalgunj Medical College Teaching Hospital at Nepalgunj, Nepal over a period of three years (April 2000April 2003).

510 New born admitted to the NICU during this period underwent careful clinical assessment and in suspected cases of NEC $(n=27)$ necessary investigations were done blood counts, blood culture, X-ray, ultrasound of abdomen and stool for blood.

NEC is suspected whenever abdominal signs predominate in a newborn infant with features of Septicemia. Early signs are indistinguishable from those of sepsis.

\section{RESULTS}

During the period of three years total newborns admitted in our NICU were 510. On the basis of the clinical features 27 cases were suspected as NEC. Therefore the total incidence at our centre was $5.30 \%$. $(27 / 510)$.

- More than 90\% (25) of newborns with NEC were preterm (mean 32 weeks) and nearly all babies gave history of enteral feeding of formula and some had ingested honey.

- About $66 \%$ of Newborns of NEC were male $(n=18)$

- $37 \%$ (10) cases have low APGAR score at Birth.

Clinical signs and symptoms range from mild feeding intolerance with abdominal distension to catastrophic disease with bowel perforation, peritonitis, and cardiovascular collapse. Vigilant assessment of at-risk neonates is crucial. When conservative medical management fails to halt injury, surgical intervention is often needed. Strategies to decrease the incidence and ultimately prevent NEC loom on the horizon, such as exclusive use of human breastmilk for enteral feedings and administration of probiotics.

- 6 babies (22.22\%) of NEC died because they were of Very Low Birth Weight (VLBW) and had septic shock. 